PROCEEDINGS OF THE

AMERICAN MATHEMATICAL SOCIETY

Volume 130, Number 8, Pages 2287-2294

S 0002-9939(02)06641-8

Article electronically published on March 25, 2002

\title{
APPLICATION OF THE BOREL TRANSFORM TO THE STUDY OF THE SPECTRUM OF INTEGRAL EQUATIONS WHOSE KERNELS ARE ENTIRE FUNCTIONS OF EXPONENTIAL TYPE
}

\author{
MURALI RAO AND LI-CHIEN SHEN \\ (Communicated by Juha M. Heinonen)
}

\begin{abstract}
Using the Borel transform, we study the spectrum of a class of non-compact integral operators whose kernels are of exponential type and square integrable on the real line. Our method also enables us to obtain an interesting characterization of a well-known integral equation involving the Bessel function $J_{0}$.
\end{abstract}

\section{INTRODUCTION}

The aim of this work is to study the spectrum of the integral operator

$$
T f=\int_{0}^{\infty} g(x-y) f(y) d y,
$$

where $g(x)$ belongs to the class $\operatorname{Exp}_{\tau} L^{2}$ of entire functions of exponential type $\tau$ and which are square integrable on the real line. With no loss of generality, we shall choose $\tau=1$ throughout this work.

The consideration of the above integral equation is motivated by the following special case:

$$
h(x)=\frac{1}{\pi} \int_{0}^{\infty} \frac{\sin (x-y)}{x-y} f(y) d y,
$$

where $f$ belongs to $L^{2}(R)$. This integral equation arises naturally in the following setting. Consider an incoming signal $f \in L^{2}(R)$. We record the $t \geq 0$ portion of the signal and designate it as

$$
A f=: f_{+}(t)=\left\{\begin{array}{l}
f(t) \text { if } t \geq 0 \\
0 \text { if } t<0
\end{array}\right.
$$

We perform the spectral analysis on $f$ by considering its Fourier transform $\left(f_{+}\right)$. The signal is reprocessed by discarding the portion of $\left(f_{+}\right)^{\wedge}$ with frequency $|w| \geq c$ and with no loss of generality, we choose $c=1$. The processed signal $h(t)$ can be

Received by the editors December 19, 2000.

2000 Mathematics Subject Classification. Primary 31A10, 34A25.

Key words and phrases. Borel transform, Bessel functions, conjugate indicator diagram, entire functions of exponential type, integral equation. 
recovered by inverting the Fourier transform

$$
\begin{gathered}
h(t)=\frac{1}{2 \pi} \int_{-1}^{1}\left(f_{+}\right)(w) e^{i t w} d w=\frac{1}{2 \pi} \int_{-1}^{1}\left(\int_{0}^{\infty} f(x) e^{-i x w} d x\right) e^{i t w} d w \\
=\frac{1}{\pi} \int_{0}^{\infty} \frac{\sin (t-x)}{t-x} f(x) d x .
\end{gathered}
$$

This gives the desired physical interpretation of the integral equation (1.1). In the general case, we have (see Theorem 2)

$$
h(t)=\frac{1}{2 \pi} \int_{-1}^{1} \hat{g}(w)\left(f_{+}\right)(w) e^{i t w} d w
$$

thus, the output signal $h(t)$ is the result of further modification of $\left(f_{+}\right)^{\wedge}$ by the multiplier

$$
\hat{g}(w)=\int_{-\infty}^{\infty} g(x) e^{-i x w} d x
$$

which is the Fourier transform of $g$. (According to Paley-Wiener theorem [5, p. $370], \hat{g}$ is a square integrable function and with support in $[-1,1]$.)

A similar integral equation for the operator $A_{1}$, which carries an $f \in L^{2}(R)$ to a time-limited function

$$
A_{1} f=\left\{\begin{array}{l}
f(t) \text { if }|t| \leq 1 \\
0 \text { if }|t|>1
\end{array}\right.
$$

yields the integral equation

$$
g(x)=\frac{1}{\pi} \int_{-1}^{1} \frac{\sin c(x-y)}{x-y} f(y) d y=: T_{c} f .
$$

This has been studied in great detail by Pollack and Slepian in [4] and the asymptotic formula for its largest eingenvalue as $c \rightarrow \infty$ was obtained by Fuchs [2]. However, operators $T$ and $T_{c}$ are fundamentally different. The operator $T_{c}$ is compact and its eigenfunctions are the prolate spheroidal functions, whereas the operator $T$ is non-compact; their method does not seem suited for the study of the spectrum of $T$ and we will approach the problem from the setting of Borel transform.

Before we proceed further, it is interesting to observe the following integral equation:

$$
\frac{1}{2} J_{0}(x)=\frac{1}{\pi} \int_{0}^{\infty} \frac{\sin (x-y)}{x-y} J_{0}(y) d y,
$$

where $J_{0}(x)$ is the Bessel function of order zero. It is important to point out that, since [6. p. 95]

$$
J_{0}(x) \sim \sqrt{\frac{2}{\pi x}} \sin \left(x+\frac{\pi}{4}\right)
$$

as $x \rightarrow \infty, J_{0}$ does not belong to $L^{2}(R)$.

The identity (1.3) is often derived as a special case of Sonnie's integral [6], p. 433], but we shall provide a different proof which serves to illustrate the essence of our approach. 
We first recall the Borel (or Laplace) transform of $J_{0}(x)$ [6] p. 384]: For $b>0$,

$$
\int_{0}^{\infty} e^{-x(b+i w)} J_{0}(x) d x=1 / \sqrt{1+(b+i w)^{2}} .
$$

Then,

$$
\begin{aligned}
& \int_{0}^{\infty} e^{-b x} J_{0}(x) \frac{\sin (x-y)}{x-y} d x \\
= & \frac{1}{2} \int_{-1}^{1} e^{i y w} \int_{0}^{\infty} e^{-x(b+i w)} J_{0}(x) d x d w \\
= & \frac{1}{2} \int_{-1}^{1} e^{i y w} / \sqrt{1+(b+i w)^{2}} d w .
\end{aligned}
$$

Since the integrand is absolutely integrable, the reversal of the integral is permissible. The equality (1.3) now follows from (1.4) by letting $b \rightarrow 0+$ (together with the dominated Lebesgue convergence theorem) and the well-known identity [, p. 48]:

$$
J_{0}(x)=\frac{1}{\pi} \int_{-1}^{1} e^{i x t} / \sqrt{1-t^{2}} d t .
$$

The major part of our work is to establish

Theorem 1. Let $g \in \operatorname{Exp}_{1} L^{2}$ and $\|\hat{g}\|_{\infty}<\infty$.

(a) The integral equation

$$
\int_{0}^{\infty} g(x-y) f(y) d y=\lambda f(x)
$$

has no solution $f$ in $L^{2}(R)$. Therefore, the operator $T$ has continuous spectrum only.

(b) Suppose $f$ is an entire function of exponential type $\tau, \tau \leq 1$, and its conjugate indicator diagram belongs to the vertical interval $I=[-i, i]$ on the imaginary axis. If the Borel transform of $f$ is integrable on $I$ and $T f=\lambda f$, then

$$
g=\frac{2 \lambda}{\pi} \frac{\sin x}{x} \text { and } f=c J_{0}
$$

for some constant $c$.

Hence our method gives the above interesting characterization of the integral equation (1.3).

The definitions of Borel transform, the indicator diagram and relevant properties of Borel transform are contained in Section 2, and Theorem 1 is derived in Section 3 .

\section{Reformulation of (1.5) in terms of Borel transform}

Let $f$ be an entire function of exponential type $\tau$. The Lindelof indicator function is defined as

$$
h_{f}(\theta)=\varlimsup_{r \rightarrow \infty}\left(\ln \left|f\left(r e^{i \theta}\right)\right|\right) / r .
$$


The indicator diagram of $f$ is the set

$$
\operatorname{Indgr} f=\left\{z: \operatorname{Re}\left(\bar{z} e^{i \theta}\right) \leq h_{f}(\theta)\right\} .
$$

The Borel transform of $f$ is defined as

$$
\varphi(w)=\int_{0}^{\infty} e^{-w t} f(t) d t=\sum_{n=0}^{\infty} f^{(n)}(0) / w^{n+1} \text { for } \operatorname{Re} w \geq h_{f}(0) .
$$

Then $\varphi$ is analytic in the half plane $\operatorname{Re} w>h_{f}(0)$ and $\varphi(\infty)=0$. Furthermore, $\varphi$ can be analytically continued to the exterior of the conjugate indicator diagram by the formula

$$
\varphi\left(w e^{i \theta}\right)=e^{-i \theta} \int_{0}^{\infty} e^{-w t} f\left(t e^{-i \theta}\right) d t .
$$

$f$ can be recovered from its Borel transform by the integral

$$
f(z)=\int_{\gamma} e^{w z} \varphi(w) d w
$$

where $\gamma$ is a simple curve surrounding the boundary of the conjugate indicator diagram. So there is a one-to-one correspondence between a given function and its Borel transform.

For the Bessel function $J_{0}$, its Borel transform is

$$
1 / \sqrt{1+w^{2}} \text {. }
$$

In the current work, we focus exclusively on the class $\operatorname{Exp}_{\tau} L^{2}, \tau \leq 1$. These functions are automatically bounded on the real line and their conjugate indicator diagrams are closed intervals contained in the interval $I=\{i y:-1 \leq y \leq 1\}$ and the limits

$$
\varphi_{+}(i w)=: \lim _{x \rightarrow 0^{+}} \int_{0}^{\infty} e^{-s t} f(t) d t, \quad s=x+i w, \quad x>0,
$$

and

$$
\varphi_{-}(i w)=: \lim _{x \rightarrow 0^{-}} \int_{0}^{\infty} e^{-s t} f(t) d t, \quad s=x+i w, \quad x<0 .
$$

Both exist almost everywhere and in $L^{2}$ sense. Therefore, for $f \in \operatorname{Exp}_{\tau} L^{2}$, the Borel transform $\varphi$ is analytic on the region $C-I$ and takes the values of $\varphi_{-}$and $\varphi_{+}$, respectively, on the left and right sides of the interval $I$. Moreover, we can express the Fourier transform $\hat{f}$ of $f$ as

$$
\hat{f}(w)=\varphi_{+}(i w)-\varphi_{-}(i w) .
$$

We can now reformulate the integral equation $T f=\lambda f$ in terms of the Borel transform of $f$ as follows. From (1.2),

$$
\lambda f=T f=\frac{1}{2 \pi} \int_{-1}^{1} e^{i w x} \varphi_{+}(i w) \hat{g}(w) d w .
$$

Applying the Fourier transform to the above equation, and together with (2.2), we have

$$
\lambda\left(\varphi_{+}(i w)-\varphi_{-}(i w)\right)=\lambda \hat{f}(w)=(T f)^{\gamma}(w)=\hat{g}(w) \varphi_{+}(i w)
$$

or

$$
\lambda \varphi_{-}(i w)=(\lambda-\hat{g}(w)) \varphi_{+}(i w) \quad \text { a.e. }
$$


for $|w| \leq 1$. The identity (2.3) is the desired reformulation of the integral equation (1.5) in terms of Borel transform.

The interested readers can find the proofs of the essential properties of Borel transform in [3].

\section{Proof of Theorem 1}

We begin by establishing some simple properties of the operator $T$.

Theorem 2. Let $g(z) \in \operatorname{Exp}_{1} L^{2}$ and $\|\hat{g}\|_{\infty}<\infty$. Define the integral operator

$$
T f=\int_{0}^{\infty} g(x-y) f(y) d y .
$$

Then:

(a) $T: L^{2}\left(R^{+}\right) \rightarrow \operatorname{Exp}_{1} L^{2}$ is bounded and $\|T\| \leq\|\hat{g}\|_{\infty}$, where $R^{+}=[0, \infty)$.

(b) If $\hat{g} \geq 0$ a.e., then $T \geq 0$.

(c) If $T f=0$, then $f=0$ a.e., unless $g=0$ a.e.; hence 0 cannot be an eigenvalue of $T$.

Proof. Let $f \in L^{1}\left(R^{+}\right) \cap L^{2}\left(R^{+}\right)$. Define, for all $x<0, f(x)=0$. Then

$$
\begin{gathered}
T f=\frac{1}{2 \pi} \int_{0}^{\infty} \int_{-1}^{1} \hat{g}(t) e^{i t((x-y)} d t f(y) d y \\
=\frac{1}{2 \pi} \int_{-1}^{1} \hat{g}(t) e^{i t x}\left(\int_{0}^{\infty} f(y) e^{-i t y} d y\right) d t \\
=\frac{1}{2 \pi} \int_{-1}^{1} e^{i t x} \hat{g}(t) \hat{f}(t) d t .
\end{gathered}
$$

The reversal of integrals is permissible, because $f$ belongs to $L^{1}\left(R^{+}\right)$.

From isometry of the Fourier transform $\sqrt{2 \pi}\|f\|=\|\hat{f}\|$,

$$
\|T f\|=\frac{1}{\sqrt{2 \pi}}\|\hat{g} \hat{f}\| \leq \frac{1}{\sqrt{2 \pi}}\|\hat{g}\|_{\infty}\|\hat{f}\|=\|\hat{g}\|_{\infty}\|f\| .
$$

Since $L^{1}\left(R^{+}\right) \cap L^{2}\left(R^{+}\right)$is dense in $L^{2}\left(R^{+}\right)$, from (3.2), we conclude that $\|T\| \leq$ $\|\hat{g}\|_{\infty}$.

Since, from $(3.1),(T f)^{\wedge}=\hat{g} \hat{f}$, which is square integrable with support in $[-1,1]$, $T f$ belongs to $\operatorname{Exp}_{1} L^{2}$. The conclusion (b) follows from (3.1):

$$
(T f, f)=(\hat{g} \hat{f}, \hat{f}) \geq 0,
$$

where $(f, g)$ is the usual inner product.

To establish (c), we observe that $\hat{f}$ belongs to the Hardy space of the lower half plane; therefore, on the real line, it can be zero only on a set of measure zero [1]. p. 166]. Suppose $T f=0$ for some $f$. Then, $\hat{g} \hat{f}=(T f)^{\wedge}=0$ a.e. From the above observation, we conclude that $\hat{g}=0$ a.e., and this establishes the desired conclusion.

Our main task is to show that equation (2.3) cannot hold and thereby establish that the integral equation (1.5) has no point spectrum, hence the spectrum is continuous. 
We reformulate the problem in the setting of the unit disc. To this end, we recall that the function

$$
h(z)=i(z+1 / z) / 2
$$

maps the interior of the disc $|z|<1$ conformally onto the region $C-I$ with $h(0)=\infty$ and the upper part of the circle corresponds to the right side of $I$ and the lower part of the circle goes to the left side of $I$. We now define

$$
F(z)=\varphi(h(z)) \text {. }
$$

Then $F(z)$ is analytic in $|z|<1$ with $F(0)=0$. Moreover, on the upper and the lower parts of the circle $|z|=1, F$ takes, respectively, the values of $\varphi_{+}$and $\varphi_{-}$. From (2.3),

$$
F\left(e^{-i \theta}\right)=\varphi_{-}(i w)=(1-\hat{g}(w) / \lambda) \varphi_{+}(i w)=G(\theta) F\left(e^{i \theta}\right) \quad \text { a.e. }
$$

where $G(\theta)=1-g(\cos \theta) / \lambda$ and we note that $G(-\theta)=G(\theta)$.

To prove (a) of Theorem 1 , we consider

$$
A(z)=F^{2}(z)\left(1-z^{2}\right) .
$$

Then, for $|z|=1$, writing $w=h(z)$,

$$
d w=\frac{i}{2}\left(1-z^{-2}\right) d z=-\frac{1}{2}\left(e^{i \theta}-e^{-i \theta}\right) d \theta, \quad|d w|=|\sin \theta| d \theta
$$

and

$$
\begin{gathered}
\int_{-1}^{1}\left|\varphi_{+}(i w)\right|^{2} d w+\int_{-1}^{1}\left|\varphi_{-}(i w)\right|^{2} d w=\int_{0}^{2 \pi}\left|F\left(e^{i \theta}\right)\right|^{2}|\sin \theta| d \theta \\
=\frac{1}{2} \int_{0}^{2 \pi}\left|F\left(e^{i \theta}\right)\right|^{2}\left|1-e^{i \theta}\right| d \theta
\end{gathered}
$$

Therefore, $A(z) \in H^{2}$, and hence the Fourier series of $A\left(e^{i \theta}\right)$ exists (see [5, Chapter 17] for the definition and properties of the $H^{P}$ space in the unit disc). Let the Fourier series of $A\left(e^{i \theta}\right)$ be

$$
\sum_{n=2}^{\infty} a_{n} e^{i n \theta}
$$

We note that since $F(0)=0, a_{0}=a_{1}=0$, and the Fourier series expansion of $A\left(e^{-i \theta}\right)$ is

$$
\sum_{n=2}^{\infty} a_{n} e^{-i n \theta}
$$

From (3.3)

$$
A\left(e^{i \theta}\right) G^{2}(\theta)=-e^{2 i \theta} A\left(e^{-i \theta}\right) \quad \text { a.e. }
$$

Then, from (3.7),

$$
\begin{aligned}
G^{2}(\theta) A\left(e^{-i \theta}\right) & =G^{2}(-\theta) A\left(e^{-i \theta}\right)=-e^{-2 i \theta} A\left(e^{i \theta}\right) \\
& =A\left(e^{-i \theta}\right) / G^{2}(\theta) .
\end{aligned}
$$

This implies that $G^{4}(\theta)=1$, hence

$$
A\left(e^{i \theta}\right)=-e^{2 i \theta} A\left(e^{-i \theta}\right) \text { or } A\left(e^{i \theta}\right)=e^{2 i \theta} A\left(e^{-i \theta}\right) \quad \text { a.e. }
$$


From (3.8), we see that $A\left(e^{i \theta}\right)$ and $e^{2 i \theta} A\left(e^{-i \theta}\right)$ must have the same Fourier coefficients. Thus, comparing their Fourier coefficients (using (3.5) and (3.6)), we deduce that $A=0$ and thus $F=0$. Hence, $\varphi=0$ which, in turn, implies $f=0$. This shows that $T f=\lambda f$ has no non-trivial solution.

We now proceed to prove Theorem 1(b).

Since, by assumption, the Borel transform of $f$ is required to be integrable on its indicator diagram I, we conclude that

$$
\begin{gathered}
\frac{1}{2} \int_{0}^{2 \pi}\left|F\left(e^{i \theta}\right)\right|\left|1-e^{2 i \theta}\right| d \theta=\int_{0}^{2 \pi}\left|F\left(e^{i \theta}\right)\right||\sin \theta| d \theta \\
\quad=\int_{-1}^{1}\left|\varphi_{+}(i w)\right| d w+\int_{-1}^{1}\left|\varphi_{-}(i w)\right| d w<\infty
\end{gathered}
$$

Hence $B(z)=F(z)\left(1-z^{2}\right) \in H^{1}$ and its boundary values $B\left(e^{i \theta}\right)$ belong to $L^{1}$. Let the Fourier series expansion of $B\left(e^{i \theta}\right)$ be

$$
\sum_{n=1}^{\infty} a_{n} e^{i n \theta} .
$$

We note that since $F(0)=0, a_{0}=0$.

From (3.3),

$$
B\left(e^{-i \theta}\right)=-B\left(e^{i \theta}\right) e^{-2 i \theta} G(\theta) \quad \text { a.e. }
$$

Then,

$$
B\left(e^{i \theta}\right)=-B\left(e^{-i \theta}\right) e^{2 i \theta} G(\theta)=B\left(e^{i \theta}\right) G^{2}(\theta)
$$

and we deduce $G^{2}(\theta)=1$. Thus, either

$$
B\left(e^{-i \theta}\right)=e^{-2 i \theta} B\left(e^{i \theta}\right) \text { or } B\left(e^{-i \theta}\right)=-e^{-2 i \theta} B\left(e^{i \theta}\right) \quad \text { a.e. }
$$

Comparing the Fourier coefficients of both sides of (3.10) (using (3.9), we obtain $a_{n}=0$ for all $n>1$ and if $a_{1} \neq 0, B(z)=a_{1} z$. This implies

$$
F(z)=a_{1} z /\left(1-z^{2}\right) .
$$

From the relation $F(z)=\varphi(h(z))$, we conclude

$$
\varphi(w)=c / \sqrt{1+w^{2}} \quad\left(c=a_{1} / 2 i\right),
$$

which, according to (2.1), is the Borel transform of $c J_{0} \cdot G^{2}(\theta)=1$ implies that $\hat{g}=2 \lambda$ or $\hat{g}=0$. This yields respectively,

$$
g=\frac{2 \lambda}{\pi} \frac{\sin x}{x}
$$

and $g=0$ for the trivial case. This establishes the desired conclusion.

We remark that the argument relies on the representation of the boundary values of the given function in terms of the Fourier series expansion. It is essential that the boundary values of the functions are in the class of $L^{p}$ for $p \geq 1$. 


\section{REFERENCES}

1. H. Dym and H.P. McKean, Fourier Series and Integral, Academic Press, New York, 1972. MR 56:945

2. W.H.J. Fuchs, On the eigenvalues of an integral equation arising in the theory of band limited signals, J. Math. Anal. Appl. 9(1964), 317-330. MR 30:4128

3. B. Ja. Levin, Distribution of Zeros of Entire Functions, Amer. Math. Soc., Providence, R.I., 1964. MR 28:217] Russian transl. MR 81k:30011

4. H.O. Pollack and D. Slepian, Prolate spheroidal wave functions Fourier analysis and uncertainty (1), Bell System Tech. J. 40(1961), 43-64.

5. W. Rudin, Real and Complex Analysis, McGraw-Hill, New York, 1966. MR 35:1420

6. G.N. Watson, A Treatise on the Theory of Bessel Functions, second edition, Cambridge University Press, London 1962. MR 6:64a; reprint MR 96i:33010

Department of Mathematics, University of Florida, Gainesville, Florida 32611

E-mail address: rao@math.ufl.edu

Department of Mathematics, University of Florida, Gainesville, Florida 32611

E-mail address: shen@math.ufl.edu 